\title{
Corrigendum: Unmet Psychosocial Needs of Patients Newly Diagnosed with Ulcerative Colitis: Results from the Nationwide Prospective Cohort Study in Korea
}

Jung Rock Moon ${ }^{1}$, Chang Kyun Lee ${ }^{1}$, Sung Noh Hong², Jong Pil Im³ ${ }^{3}$ Byong Duk Ye', Jae Myung Cha ${ }^{5}$, Sung-Ae Jung $^{6}$, Kang-Moon Lee ${ }^{7}$, Dong II Park ${ }^{8}$, Yoon Tae Jeen ${ }^{9}$, Young Sook Park ${ }^{10}$, Jae Hee Cheon ${ }^{11}$, Hyesung Kim ${ }^{12}$, BoJeong $\mathrm{Seo}^{12}$, Youngdoe $\mathrm{Kim}^{12}$, and Hyo Jong $\mathrm{Kim}^{1}$, the MOSAIK study group of the Korean Association for the Study of Intestinal Diseases (KASID)

${ }^{1}$ Center for Crohn's and Colitis, Department of Gastroenterology, Kyung Hee University College of Medicine, ${ }^{2}$ Department of Medicine, Sungkyunkwan University School of Medicine, ${ }^{3}$ Department of Internal Medicine, Seoul National University College of Medicine, ${ }^{4}$ Department of Gastroenterology, University of Ulsan College of Medicine, ${ }^{5}$ Department of Internal Medicine, Kyung Hee University Hospital at Gangdong, ${ }^{6}$ Department of Internal Medicine, Ewha Womans University College of Medicine, ${ }^{7}$ Department of Internal Medicine, College of Medicine, The Catholic University of Korea, ${ }^{8}$ Department of Internal Medicine, Kangbuk Samsung Hospital, Sungkyunkwan University School of Medicine, ${ }^{9}$ Department of Gastroenterology and Hepatology, Korea University College of Medicine, ${ }^{10}$ Department of Internal Medicine, Eulji University School of Medicine, ${ }^{11}$ Department of Internal Medicine, Yonsei University College of Medicine, and ${ }^{12}$ Medical Affairs, Janssen Korea, Seoul, Korea

https://doi.org/10.5009/gnl19107

Gut Liver 2020;14(4):459-467

In the version of this article initially published, Table 2 contains an error in the data of p-value.

On page 462, the table should be corrected as follows (marked in bold):

From:

Table 2. Mean Values of Each Patient-Reported Outcome Measure

\begin{tabular}{|c|c|c|c|c|c|c|}
\hline \multirow{2}{*}{ PROs } & \multirow{2}{*}{ Category } & \multirow{2}{*}{ No. } & \multirow{2}{*}{ Overall ( $n=355$ ) } & \multicolumn{2}{|c|}{ Disease severity } & \multirow{2}{*}{$p$-value } \\
\hline & & & & Moderate $(n=330)^{*}$ & Severe $(n=25)^{\dagger}$ & \\
\hline \multirow[t]{2}{*}{ HADS } & Depression & 349 & $6.6 \pm 4.5$ & $6.5 \pm 4.4$ & $8.9 \pm 5.2$ & 0.610 \\
\hline & Anxiety & 348 & $6.2 \pm 4.4$ & $6.2 \pm 4.4$ & $6.6 \pm 4.5$ & 0.638 \\
\hline \multirow[t]{4}{*}{ WPAI:UC } & Absenteeism ${ }^{\ddagger}$ & 149 & $29.1 \pm 36.9$ & $27.3 \pm 35.8$ & $54.1 \pm 43.5$ & 0.102 \\
\hline & Presenteeism ${ }^{\S}$ & 143 & $37.8 \pm 31.4$ & $36.8 \pm 30.3$ & $62.2 \pm 39.6$ & 0.037 \\
\hline & Overall work impairment" & 130 & $45.5 \pm 32.7$ & $44.1 \pm 31.9$ & $70.7 \pm 37.8$ & 0.048 \\
\hline & Social activity impairment $t^{\rrbracket}$ & 344 & $46.4 \pm 31.2$ & $45.2 \pm 31.0$ & $61.2 \pm 31.0$ & 0.014 \\
\hline \multirow[t]{5}{*}{ IBDQ } & Bowel system & 344 & $41.7 \pm 13.0$ & $42.0 \pm 13.1$ & $36.1 \pm 10.9$ & 0.027 \\
\hline & Emotional health & 347 & $51.7 \pm 14.9$ & $52.0 \pm 14.9$ & $46.8 \pm 13.6$ & 0.097 \\
\hline & Systemic symptoms & 347 & $20.5 \pm 6.8$ & $20.7 \pm 6.8$ & $17.0 \pm 6.0$ & 0.009 \\
\hline & Social function & 309 & $23.2 \pm 7.7$ & $22.8 \pm 7.9$ & $18.0 \pm 6.8$ & 0.003 \\
\hline & Total score & 305 & $137.3 \pm 38.8$ & $137.5 \pm 39.0$ & $117.9 \pm 33.2$ & 0.002 \\
\hline
\end{tabular}

Data are presented as the mean \pm SD.

PRO, patient-reported outcome; HADS, Hospital Anxiety and Depression Scale; WPAl, Work Productivity and Activity Impairment; UC, ulcerative colitis; IBDQ, Inflammatory Bowel Disease Questionnaire.

${ }^{*}$ Total Mayo score 6-10 points; ${ }^{\dagger}$ Total Mayo score $\geq 11$; ${ }^{\ddagger}$ Absenteeism: percentage of work time missed=[Q2/[Q2+Q4]]) $\times 100 ;{ }^{\S}$ Presenteeism: percent-

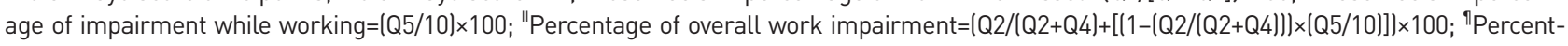
age of social activity impairment=(Q6/10) $\times 100 ; Q$, questionnaire item. 
To:

Table 2. Mean Values of Each Patient-Reported Outcome Measure

\begin{tabular}{|c|c|c|c|c|c|c|}
\hline \multirow{2}{*}{ PROs } & \multirow{2}{*}{ Category } & \multirow{2}{*}{ No. } & \multirow{2}{*}{ Overall ( $n=355$ ) } & \multicolumn{2}{|c|}{ Disease severity } & \multirow{2}{*}{$\mathrm{p}$-value } \\
\hline & & & & Moderate $(n=330)^{*}$ & Severe $(n=25)^{\dagger}$ & \\
\hline \multirow[t]{2}{*}{ HADS } & Depression & 349 & $6.6 \pm 4.5$ & $6.5 \pm 4.4$ & $8.9 \pm 5.2$ & 0.024 \\
\hline & Anxiety & 348 & $6.2 \pm 4.4$ & $6.2 \pm 4.4$ & $6.6 \pm 4.5$ & 0.638 \\
\hline \multirow[t]{4}{*}{ WPAI:UC } & Absenteeism ${ }^{\ddagger}$ & 149 & $29.1 \pm 36.9$ & $27.3 \pm 35.8$ & $54.1 \pm 43.5$ & 0.102 \\
\hline & Presenteeism ${ }^{\S}$ & 143 & $37.8 \pm 31.4$ & $36.8 \pm 30.3$ & $62.2 \pm 39.6$ & 0.037 \\
\hline & Overall work impairment" & 130 & $45.5 \pm 32.7$ & $44.1 \pm 31.9$ & $70.7 \pm 37.8$ & 0.048 \\
\hline & Social activity impairment ${ }^{\Uparrow}$ & 344 & $46.4 \pm 31.2$ & $45.2 \pm 31.0$ & $61.2 \pm 31.0$ & 0.014 \\
\hline \multirow[t]{5}{*}{ IBDQ } & Bowel system & 344 & $41.7 \pm 13.0$ & $42.0 \pm 13.1$ & $36.1 \pm 10.9$ & 0.027 \\
\hline & Emotional health & 347 & $51.7 \pm 14.9$ & $52.0 \pm 14.9$ & $46.8 \pm 13.6$ & 0.097 \\
\hline & Systemic symptoms & 347 & $20.5 \pm 6.8$ & $20.7 \pm 6.8$ & $17.0 \pm 6.0$ & 0.009 \\
\hline & Social function & 309 & $23.2 \pm 7.7$ & $22.8 \pm 7.9$ & $18.0 \pm 6.8$ & 0.003 \\
\hline & Total score & 305 & $137.3 \pm 38.8$ & $137.5 \pm 39.0$ & $117.9 \pm 33.2$ & 0.002 \\
\hline
\end{tabular}

Data are presented as the mean \pm SD.

PRO, patient-reported outcome; HADS, Hospital Anxiety and Depression Scale; WPAI, Work Productivity and Activity Impairment; UC, ulcerative colitis; IBDQ, Inflammatory Bowel Disease Questionnaire.

*Total Mayo score 6-10 points; ${ }^{\dagger}$ Total Mayo score $\geq 11 ;{ }^{\ddagger}$ Absenteeism: percentage of work time missed=(Q2/[Q2+Q4]) $\times 100 ;{ }^{\S}$ Presenteeism: percentage of impairment while working=(Q5/10) $\times 100$; "Percentage of overall work impairment=(Q2/(Q2+Q4)+[(1-(Q2/(Q2+Q4))) $\times(Q 5 / 10)]) \times 100$; ${ }^{1} P e r c e n t-$ age of social activity impairment=(Q6/10) $\times 100 ; Q$, questionnaire item. 